This is an author-created, un-copyedited version of an article published in Journal of Physics: Condensed Matter. IOP Publishing Ltd is not responsible for any errors or omissions in this version of the manuscript or any version derived from it. The Version of Record is available online at http://dx.doi.org/10.1088/0953-8984/25/32/325101.

\title{
The Self-Assembly of Particles with Isotropic Interactions
}

\author{
K. von Konigslow, E. D. Cardenas-Mendez, and R. B. Thompson* \\ Department of Physics and Astronomy, \\ University of Waterloo, 200 University Avenue West, \\ Waterloo, Ontario, Canada N2L $3 G 1$ and \\ Waterloo Institute for Nanotechnology, \\ University of Waterloo, 200 University Avenue West, \\ Waterloo, Ontario, Canada N2L 3G1
}

\author{
K. Ø. Rasmussen \\ Theoretical Division, Los Alamos National Laboratory, \\ Los Alamos, New Mexico, USA 87545
}

\begin{abstract}
A generic field-theoretic model for the self-assembly of particles with isotropic interactions, motivated by ideas in DNA-mediated colloidal assembly, is presented. A simplest possible system of colloids in explicit solvent is examined to determine the ability of non-connected particles to form complex nanometre or micron scale equilibrium structures in the absence of confounding kinetic effects. It is found that non-trivial morphologies are possible and that, for this effectively one component system, these parallel the phases of diblock copolymer melts for certain parameter choices, despite the absence of connectivity or packing frustration in the model. An explanation for the morphological similarity between these architecturally disparate systems is given. For other parameter choices, it is found that meta-stable and defected phases become more common, and that similarity with block copolymer morphologies decreases.
\end{abstract}

\footnotetext{
${ }^{*}$ Electronic address: thompson@uwaterloo.ca
} 


\section{INTRODUCTION}

DNA-mediated colloidal organization is one of the newest concepts in self-assembly. First introduced by Mirkin et al. [1] and Alivisatos et al. [2], the field has grown rapidly with contributions by the groups of Gang [3], Crocker [4], Frenkel [5, 6], Chaiken [7-9], Pine [7, 8, 10], Starr [11] and others [12-15]. In DNA coated colloid (DNACC) systems, colloids are covered with DNA molecules such that selective and mutually exclusive interactions can take place depending on the coatings. Through this preparation, the ultimate goal is to be able to construct, through self-assembly, pre-determined three dimensional nanometre and micron length scale structures. For a succinct review of DNACCs, the reader is referred to the article by Di Michele and Eiser [16]. To gain insight into this type of system, Tindemans and Mulder used a philosophy of ignoring the important, but system-specific, kinetic DNA bonding issues and focused instead on generic equilibrium structures of selectively and isotropically interacting particles in a 2D lattice-based approach [17]. From this perspective, DNAmediated colloids are to a first approximation isotropic [18] and are just one example of a class of selectively and isotropically interacting potentials that also includes single species colloids with two length-scale interactions $[19,20]$ and self-assembling polymer stabilized colloids [21, 22]. For this class of self-assembly, Manoharan has suggested the concept of larger scale assembly, where building blocks are given tailored interactions that will cause them to form desired objects [23]. Frenkel and Wales suggest two important design rules for any generic system with selectively and isotropically interacting potentials: structures should be kinetically accessible, and structures should reach thermodynamic equilibrium [5].

In this paper, we are interested in discovering if a minimalist field-theoretic model of selectively interacting particles with isotropic interactions can be expected to robustly form stable microphase separated phases, and if so, to what degree they are similar to DNACC or polymer stabilized colloids. Important information about complicated systems including water, glasses and fluid-fluid phase transitions has been revealed using soft-core isotropic potentials, even when there is anisotropy in the non-coarse grained molecular potentials

- see the review by Buldyrev et al. [19]. If the potentials are selective and have two length-scales, frustration can in principle be created which can lead to nanometre or micron scale patterns, although this is not assured. It may be that no window of microphase separation will exist between macrophase separation and uniform mixing. For example, 
DNACC systems will often assemble only in extremely narrow windows of temperature [16]. Although experiment and simulations show self-assembly [19, 21], it is not definitively known if this organization is truly equilibrium. If one is not able to form equilibrium structures in a highly idealized generic model, it may not be reasonable to expect DNA colloids, with their kinetically constraining strong specific bonding, to do so. Should DNACC structures turn out to be limited to non-equilibrium assemblies, and so violate the second design rule suggested by Frenkel and Wales, predictive engineering of their self-assembly would be much more challenging and their usefulness could be significantly curtailed. If on the other hand, self-assembly in a generic model is robust, it may be that many different species, or overly complicated inter-particle potentials are required to form diverse and useful structures. Again, the more involved DNACC case would be even more daunting, potentially limiting its utility.

It would seem then that a good step in determining the future usefulness of DNA-mediated colloidal self-assembly, or related systems, is to investigate generic and kinetically simpler systems as a limiting case. In particular, a method in which equilibrium behaviours can be immediately discovered would be valuable in benchmarking such a generic model against the second design rule of Frenkel and Wales. Self-consistent field theory (SCFT) has played just such a role in the field of block copolymer self-assembly [24-26]. In this work, we present a new SCFT suitable for investigations of the equilibrium behaviour of isotropically interacting colloids. To proceed methodically, we introduce this approach for perhaps the simplest possible case, that of a single type of colloid in a suspension. In order to create the frustration necessary for possible self-assembly, we give the isotropic interactions between colloids two length-scales, one that is short range attractive, the other longer range repulsive. Such potentials have been shown to cause microphase separation under certain conditions [27, 28]. Excluded volume interactions are treated separately through an incompressibility constraint. This model is therefore similar to colloids coated with palindromic sequences of DNA, that is, with complementary strands on the same colloid, in that it deals with aggregation in a single colloid component system [16]. It is also similar to self-assembling polymer stabilized colloids $[21,22]$, although again in a generic sense [29]. Nonetheless, the same class of self-assembly is under investigation and the formalism can be easily extended to include more species, mapping it more closely to other DNACC cases if desired. Here, to understand the important physics, the model is made as minimalist as possible; kinetics and strong bonding issues 
related to some DNA-colloid self-assembly are not addressed. In particular, colloid excluded volume interactions are treated only in an ensemble averaged way, meaning that the details of how colloids pack on colloid length scales are not treated. Gross, qualitative features will nonetheless be apparent, which is sufficient to answer preliminary questions about the ability of these systems to self-assemble into useful structures. The present approach is therefore intended to be complementary to simulation methods that are well suited to studying many aspects of DNACC systems [16].

It is found that self-assembly can be robust in three dimensions in certain cases, specifically when the ratio of the explicit solvent molecular volume to the colloid particle volume is not too small. This is a necessary, although not sufficient, condition for robust DNA colloidal self-assembly. Surprisingly, the model system self-assembles to the same qualitative phases as diblock copolymer melts, despite possessing neither packing frustration nor connectivity of chemical species. When the solvent to colloid volumetric ratio is decreased, similarities with diblock phases decrease and remaining stable phases become more difficult to achieve. Specifically, more defects appear, reflecting numerous meta-stable states, and parameters related to temperature have to be given extreme values to stabilize even these morphologies.

\section{THEORY}

The model consists of a system of two particle species, referred to as A and B. Species A represents the colloids of interest and species B is an explicit solvent which, together with an incompressibility constraint, provides an ensemble average excluded volume. The volume ratio between $\mathrm{A}$ and $\mathrm{B}$ type particles is given by $\alpha=v_{B} / v_{A}$. We define $v_{A} \equiv v_{0}$ to be a reference volume and $\sigma=\sqrt[3]{v_{0}}$ as the length scale through which all distances are made dimensionless. Thus $v_{0} n_{A}=f_{A} V$, where $n_{A}$ is the total number of A particles, $f_{A}$ is the total volume fraction of A particles, and $V$ is the system volume. Because the system is incompressible, one can, without loss of generality, choose a single potential to completely describe all pair interactions of this system. It is convenient to choose this as the effective interactions between $A$ colloids, $U_{A A}(r)$, which to be isotropic, is a function only of the radial separation between colloids. This potential, together with an energy that quadratically penalizes deviations from incompressibility, can be used to write the canonical partition function for this system. This partition function can be converted using 
Hubbard-Stratonovich transformations to a field based representation using a path integral formalism. This derivation parallels SCFT for block copolymers and details for it can be found elsewhere [26]. A saddle function approximation is performed on the resulting path integral partition function - which is equivalent to a mean field approximation - to give a free energy functional. Expressing this as a dimensionless free energy density, using the thermal energy $k_{B} T$ and the reference volume $v_{0}$, gives

$$
\begin{aligned}
\frac{v_{0} F}{k_{B} T V}= & -f_{A} \ln \left(\frac{v_{0} Q_{A}}{V f_{A}}\right)-\frac{f_{B}}{\alpha} \ln \left(\frac{v_{0} Q_{B} \alpha}{V f_{B}}\right) \\
& +\frac{1}{2} \frac{v_{0}}{V} \int \frac{d \mathbf{r}}{v_{0}}\left\{\int \frac{d \mathbf{r}^{\prime}}{v_{0}} \varphi_{A}(\mathbf{r}) \frac{U_{A A}\left(\left|\mathbf{r}-\mathbf{r}^{\prime}\right|\right)}{k_{B} T} \varphi_{A}\left(\mathbf{r}^{\prime}\right)\right. \\
& \left.-w_{A}(\mathbf{r}) \varphi_{A}(\mathbf{r})-w_{B}(\mathbf{r}) \varphi_{B}(\mathbf{r})+\frac{\kappa}{2}\left(\varphi_{A}(\mathbf{r})+\varphi_{B}(\mathbf{r})-1\right)^{2}\right\}
\end{aligned}
$$

where $Q_{A}$ and $Q_{B}$ are single particle partition functions subject to fields $w_{A}(\mathbf{r})$ and $w_{B}(\mathbf{r})$, respectively, that are defined as

$$
\begin{aligned}
Q_{A} & =\int \frac{d \mathbf{r}}{v_{0}} e^{-w_{A}(\mathbf{r})} \\
Q_{B} & =\int \frac{d \mathbf{r}}{v_{0}} e^{-\alpha w_{B}(\mathbf{r})}
\end{aligned}
$$

and $\kappa$ is a parameter that is chosen sufficiently large so as to enforce incompressibility. The four functions in (1) are the spatially dependent volume fractions $\varphi_{A}(\mathbf{r})$ and $\varphi_{B}(\mathbf{r})$ of $A$ colloids and $B$ solvent, respectively, and the conjugate chemical potential fields $w_{A}(\mathbf{r})$ and $w_{B}(\mathbf{r})$ that are the mean fields felt by A and B particles. Minimizing the free energy with respect to the volume fraction and field functions yields self-consistent equations:

$$
\begin{aligned}
\varphi_{A}(\mathbf{r}) & =\frac{f_{A} V}{v_{0} Q_{A}} e^{-w_{A}(\mathbf{r})} \\
\varphi_{B}(\mathbf{r}) & =\frac{f_{B} V}{v_{0} Q_{B}} e^{-\alpha w_{B}(\mathbf{r})} \\
w_{A}(\mathbf{r}) & =\int \frac{d \mathbf{r}^{\prime}}{v_{0}} \varphi_{A}\left(\mathbf{r}^{\prime}\right) \frac{U_{A A}\left(\left|\mathbf{r}-\mathbf{r}^{\prime}\right|\right)}{k_{B} T}+\kappa\left(\varphi_{A}(\mathbf{r})+\varphi_{B}(\mathbf{r})-1\right) \\
w_{B}(\mathbf{r}) & =\kappa\left(\varphi_{A}(\mathbf{r})+\varphi_{B}(\mathbf{r})-1\right) .
\end{aligned}
$$

The potential $U_{A A}(r)$ is chosen to be isotropic, differentiable over its domain, and to be short range attractive and longer range repulsive. A simple piecewise function that qualitatively meets these criteria is

$$
\frac{U_{A A}(r)}{k_{B} T}=\left\{\begin{array}{cc}
-\frac{\left(A_{1}+A_{2}\right)}{2} \cos \left(\frac{\pi r}{\lambda}\right)-\frac{\left(A_{1}-A_{2}\right)}{2} & , r \leq \lambda \\
A_{2} \exp \left(-\frac{(r-\lambda)^{2}}{2 \gamma^{2}}\right) & , r>\lambda
\end{array}\right.
$$




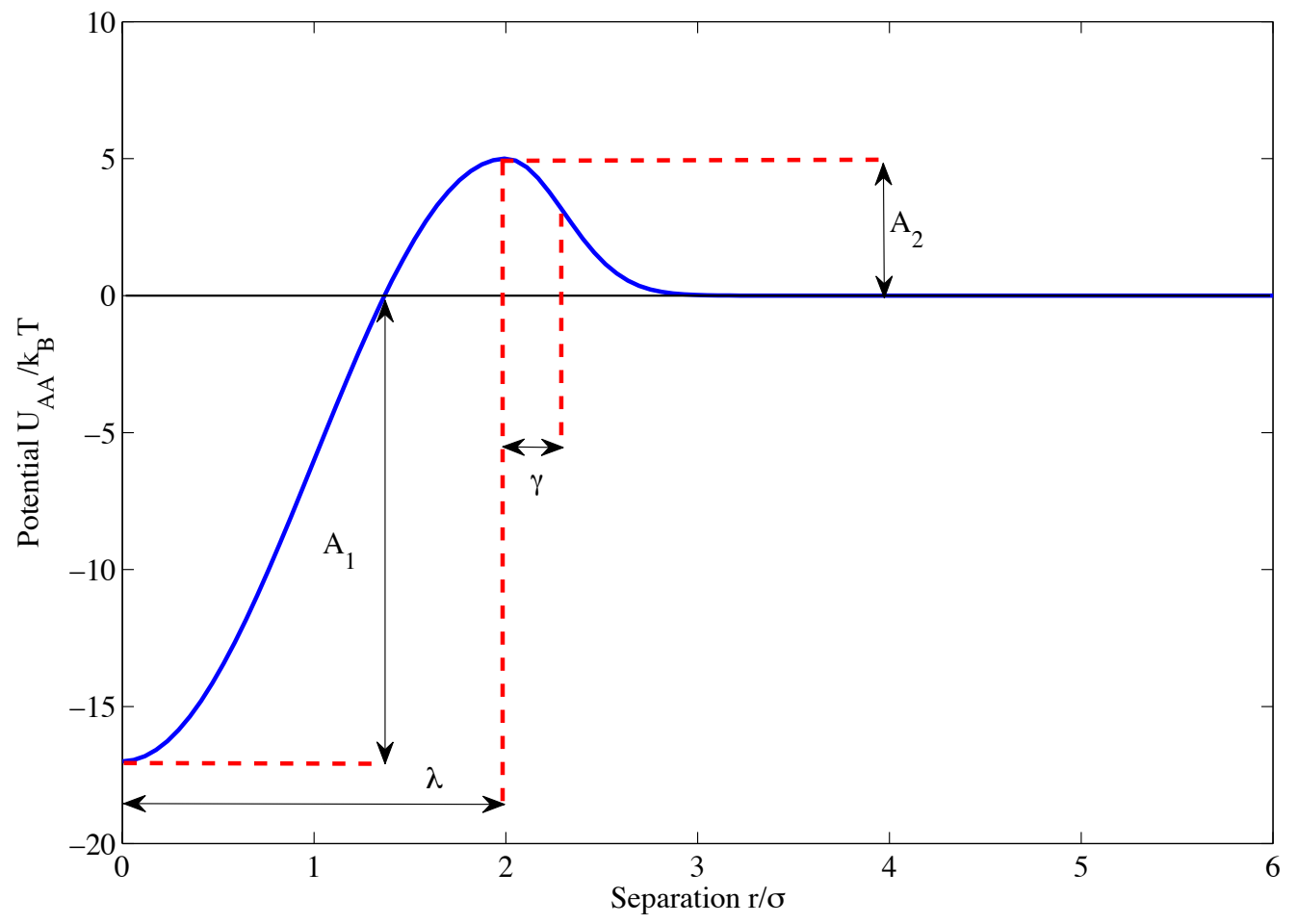

FIG. 1: Radial pair potential between A colloids chosen to be smooth, isotropic and to have a short range attraction and longer range repulsion.

where $A_{1}$ and $A_{2}$ give the depth and height of the attractive and repulsive portions of the potential, respectively, $\lambda$ gives the width of the potential well, and $\gamma$ controls the width of the potential barrier - see figure 1. The use of a short range attractive and long range repulsive potential is not an immediately intuitive choice in the present context. First, there must of course be a shortest range repulsion representing the excluded volume of the colloids or the compression of DNA coatings. In this model, this is separate from the above potential and is instead incorporated via the incompressibility constraint which enforces an ensemble averaged excluded volume. Next, the short range attraction corresponds to colloid van der Waals interactions in the case of polymer grafted colloids, or DNA mediated attraction in the case of DNA coated colloids. Such an attractive pair potential has been measured by Crocker and co-workers $[30,31]$. Finally, the longer range repulsion corresponds to the polymer entropic penalty in the case of polymer grafted colloids, and to the saturation of complementary ends in one component DNA coated colloids. In the latter case, an aggregate of larger size results, representing a new length scale of soft excluded volume. 
Strictly speaking, pair interactions are not sufficient for a description of coated nano-sized particles and many-body effects should be incorporated for a quantitative description [16]. This is obviously not a concern here however, due to the coarse nature of the present model and the qualitative approach taken. In other words, the pair potential given above can be viewed as a phenomenological effective pair interaction.

In all, this model has six physical parameters related to the potential and the macroscopic features of the system. These parameters are the total volume fraction of colloids $f_{A}$, the solvent-to-colloid volume ratio $\alpha$, the depth of the potential well $A_{1}$, the height of the potential barrier $A_{2}$, the width of the potential well $\lambda$ and the width of the potential barrier $\gamma$. The temperature $k_{B} T$ has been scaled out of the potential so that the magnitudes of $A_{1}$ and $A_{2}$ are inversely proportional to temperature. Since we want the model to represent generic colloids (A species) in a suspension (B species), we want the B particles to be much smaller than the A particles. We qualitatively accomplished this by choosing $0.5 \geq \alpha \geq 0.01$ as this range of $\alpha$ converged readily in three dimensional calculations. For quantitative realism, the value should obviously be much smaller still, but these values are acceptable for a preliminary qualitative analysis [32]. We found that non-trivial (microphase separated) phases could be produced for this choice when we fixed $\lambda=2$ and $\gamma=0.55$ for the A pair potential together with either $A_{1}=11, A_{2}=3$ or $A_{1}=3, A_{2}=0.3$, depending on the smallness of $\alpha$. Different choices of $\lambda$ and $\gamma$, were tried, but resulted in no qualitative differences. Larger changes caused either macrophase separation or a lack of convergence. Since $\lambda$ and $\gamma$ are on the order of the particle size $\sigma$, the $A$ particles should be considered as nano-sized objects. Strong bonding effects are not considered in this work, so addressing nanoscale colloids is appropriate since they generally have weaker interactions due to hosting fewer DNAs on their surfaces [16]. Changing $A_{1}$ and $A_{2}$ corresponds to changing the temperature, and we find that larger values of $A_{1}$ and $A_{2}$ (lower temperatures) are required to stabilize morphologies with smaller $\alpha$. In addition to the six physical parameters, there is the incompressibility parameter $\kappa$ and the system volume $V$ which are chosen for computational expediency. Specifically, equations (2)-(7) were solved numerically using Picard iteration on the volume fraction functions using a $\kappa$ large enough to enforce excluded volume to within less than one percent on average and volumes $V$ of 8, 10, and $15 \sigma$ units per cubic side to establish, with reasonable certainty, the morphology of a given point of parameter space. Further details concerning the theory and computational approach can be found in references 33 . 


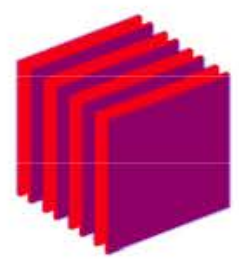

(a) Lamella

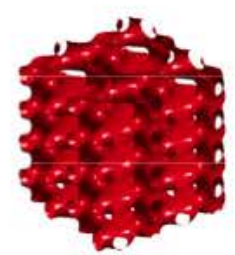

(b) Gyroid

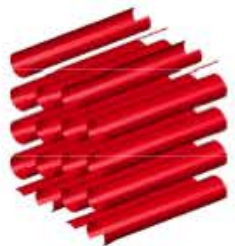

(c) Cylinders

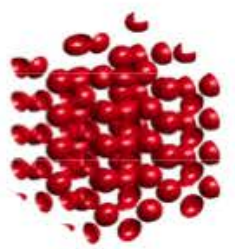

(d) Spheres

FIG. 2: The progression of phases, for $\alpha=0.5$, observed as $f_{A}$ is decreased from $f_{A}=0.5$. From $f_{A}=0.5$ to $f_{A} \approx 0.45$, lamellae $(\mathrm{L})$ are observed $(\mathrm{a})$. Near $f_{A} \approx 0.4$, the system moves to a bicontinuous gyroid phase (b). From $f_{A} \approx 0.35$ to $f_{A} \approx 0.25$, the system enters a hexagonally packed cylindrical phase $(\mathrm{C})$ phase $(\mathrm{c})$. From $f_{A} \approx 0.2$ to $f_{A} \approx 0.15$, the system enters a body centred cubic spherical $(\mathrm{S})$ phase $(\mathrm{d})$, before finally becoming disordered as $f_{A}<0.15$. This result parallels the progression of phases for diblock copolymer melts while varying the same parameter. Note that these results were computed using an $L=8 \sigma$ sided box. The results have been tiled for clarity.

\section{RESULTS AND DISCUSSION}

Figure 2 shows typical morphologies found for $A_{1}=3, A_{2}=0.3, \lambda=2, \gamma=0.55$ and $\alpha=0.5$ for a variety of $f_{A}$ volume fractions. For $f_{A}<0.15$, we found homogeneous, structureless (disordered) morphologies. We did not calculate above $f_{A}=0.5$ since we are assuming the colloids are not the majority component. We observed the standard classical phases, namely lamellar (L), hexagonally packed cylindrical (C) and body-centered cubic spheres (S). In addition, we found complex phases between the $\mathrm{L}$ and $\mathrm{C}$ domains. These complex phases seem to be gyroid $(\mathrm{G})$ and perforated lamellar (PL). Overall, this sequence of phases parallels that of diblock copolymers [24], which is remarkable given the radical difference in molecular architecture between copolymers and the present model. In the case of diblock copolymers, it is packing frustration that determines the stable phase through the competition between macromolecular chain stretching and interfacial tension [24, 34]. Although both the present system and the diblock system have binary chemical species, there can be no chain stretching in our colloidal model since the constituents are not polymers. Amphiphilic systems without chain degrees of freedom have recently been studied by one of us [35] and this revealed that standard diblock phases are not found when chain stretching is 
absent. It is not just that chain stretching is absent in the present model however, but that now there is no longer any connectivity between the two chemical species. The similarity to diblock copolymer phases can be understood through the two length-scale potential, which is apparently mimicking the competition between chain stretching and energy penalties. The A pair potential, which is long range repulsive and short range attractive, is equivalent in an incompressible system to an $\mathrm{AB}$ interaction that is long range attractive and short range repulsive. This then mirrors the diblock copolymer case, wherein AB monomers are effectively repelled at short distances, as expressed by the Flory-Huggins interaction parameter, and "attracted" at longer distances due to chain connectivity and stretching.

This example of diblock-like phases in the colloid system, shown in figure 2, is for an artificial ratio of $\alpha=0.5$ between the volume of a solvent molecule and the volume of a colloid. Figures 3 and 4 show results of various $f_{A}$ volume fractions for $\alpha$ values between 0.1 to 1.0 and 0.01 to 0.1 , respectively. As the $\alpha$ ratio gets smaller, even though still far from realistic values [32], one observes that the self-assembled morphologies no longer map so nicely to the block copolymer case. It was found to be more difficult to find the complex phases, and defects or meta-stable results occurred more frequently. The results shown in figures 3 and 4 are sensitive to the parameters used in the potential $U_{A A}(r)$ as can be seen by comparing $\alpha=0.1$ between the two maps. For figure 3, no self-assembled morphologies are found while for figure 4 self-assembly is observed. For smaller values of $\alpha$, larger magnitudes for the potential were required to achieve self-assembly. This is logical given that small values of $\alpha$ correspond to small solvent molecules with concomitant large translational entropy. Strong potentials corresponding to lower temperatures are required to overcome this large entropy in order to form non-trivial phases. Extreme values of the parameters led to numerical issues which limited the smallness of $\alpha$ that was accessible in this preliminary work. Therefore, the lack of self-assembly for $\alpha=0.01$ in figure 4 does not mean that self-assembly is not possible, merely that it was not practical to examine it in this study. A qualitative trend however seems detectable, namely that as $\alpha$ becomes small, the classical phases dominate, with defects and meta-stable states becoming more common. This is not inconsistent with the results of [21] for polymer stabilized colloids, in which spheres, cylinders, strings and sheets were observed in aggregations of polymer grafted nano-particles, often without long range order. This agreement exists despite the simplicity of the present model and the anisotropy of the interactions postulated between 


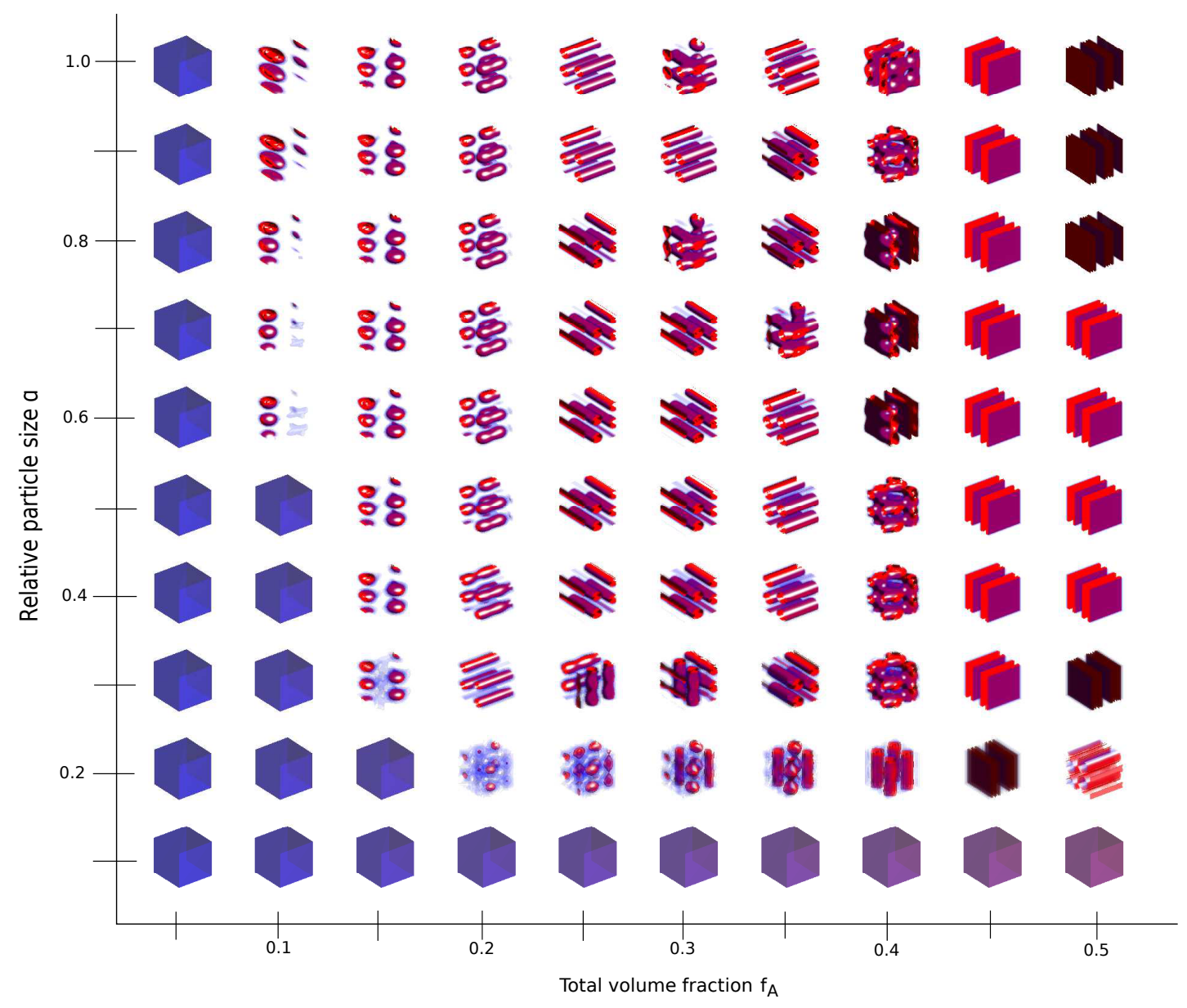

FIG. 3: Phase map for $\alpha$ between 0.1 and 1.0 for various $f_{A}$ values. Figure 2 is a blow up of a slice through $\alpha=0.5$. Isosurfaces show blue facing the $A$ colloid domains and red facing the $B$ solvent matrix. Disordered regions appear as blank blue-reddish phases. Parameters for the $U_{A A}$ potential are $A_{1}=3, A_{2}=0.3, \lambda=2$ and $\gamma=0.55$.

particles for the experimental system.

\section{CONCLUSIONS AND FUTURE DIRECTIONS}

A minimalist generic model of colloidal self-assembly is shown to produce non-trivial structures through a two length-scale, isotropic, interaction. This demonstrates, in principle, that useful equilibrium morphologies may be achieved solely through isotropic interactions, provided these interactions have competing length scales. This is consistent with theory and 


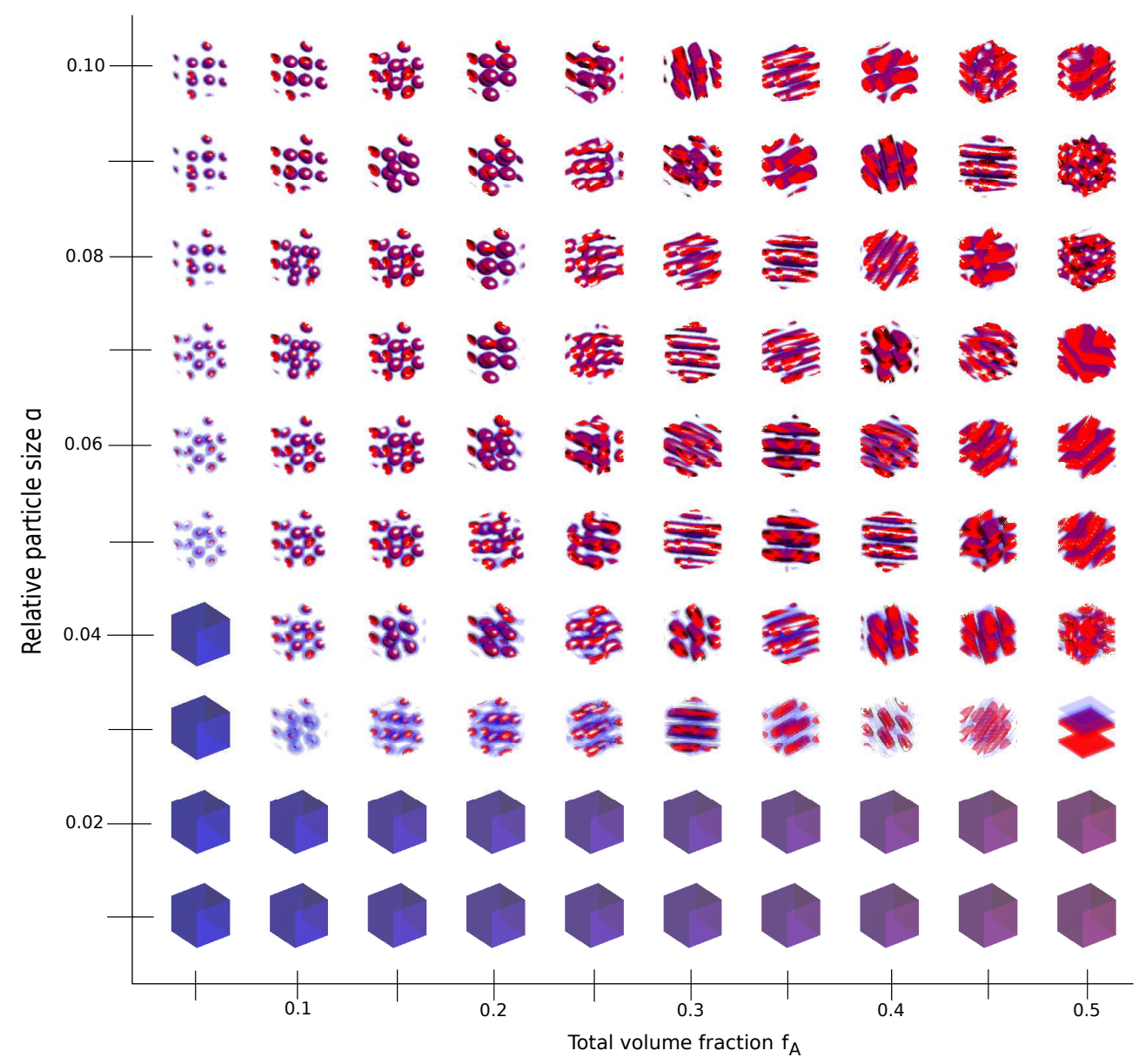

FIG. 4: Phase map for $\alpha$ between 0.01 and 0.1 for various $f_{A}$ values. Isosurfaces show blue facing the $A$ colloid domains and red facing the $B$ solvent matrix. Disordered regions appear as blank blue-reddish phases. Parameters for the $U_{A A}$ potential are $A_{1}=11, A_{2}=3, \lambda=2$ and $\gamma=0.55$.

simulations reviewed by Buldyrev et al. [19]. In reality, many interesting nanometre and micron scale structures could be created using DNA-mediated colloidal interactions, but this would require multiple types of selective interactions between the colloids. To approach a predictive capacity for such complicated systems, the present model should be extended to multiple species, using the isotropic interactions as an approximation for the selectively interacting coatings on different colloids following the philosophy of Tindemanns and Mulder [17]. To make a connection with faithful representations of DNACCs from simulations, the phenomenological potential used in this work needs to be related to physical parameters of the system. In the future, one might also consider enforcing excluded volume more rigor- 
ously by including classical density functional theory terms in the free energy. This would account for packing effects and could determine the degree to which qualitative morphologies obtained with ensemble average excluded volume are accurate. In order to begin addressing the first design rule of Frenkel and Wales [5], specifically that desired structures should be kinetically accessible, a dynamic form of this new SCFT, one that should include bonding effects, could be developed. Since the aforementioned future directions would require longer calculations, recent advances in Anderson iteration could be implemented for this new SCFT in order to speed convergence [35-38]. This, combined with an approximation related to the smallness of the volume ratio parameter $\alpha$, could allow more realistic volume ratios between colloids and solvent molecules to be studied. Achieving smaller $\alpha$ volume ratio values is an important issue for exploring the qualitative behaviour of DNA coated colloids in a more relevant regime. Also, since the main computational step for this method is merely a fast Fourier transform, these equations are well suited to being solved using GPUs.

For the present single colloid in explicit solvent model, the same phases as diblock copolymers were found. Namely, classical lamellar, hexagonally packed cylindrical and body centred cubic spherical phases, as well as complex, three point coordinated structures. This qualitative agreement arises due to the two length-scale potential which mimics packing frustration in copolymer systems. Since the present SCFT equations are so much simpler than those of a diblock copolymer system, this mapping raises the intriguing possibility of

performing inverse statistical mechanics on the simpler SCFT to find the potential required to create a given morphology. It may then be possible to use the features of this potential as a guide for choosing features for a block copolymer that could self-assemble into a similar morphology. As a first step, one could investigate a three chemical species system to see if it produces morphologies similar to ABC triblock copolymers.

\section{Acknowledgments}

Some calculations were performed on the computing facilities of SHARCNET.

[1] C. A. Mirkin, R. L. Letsinger, R. C. Mucic, and J. J. Storhoff, Nature 382, 607 (1996). 
[2] A. P. Alivisatos, K. P. Johnsson, X. Peng, T. E. Wilson, C. J. Loweth, M. P. Bruchez, and P. G. Schultz, Nature 382, 609 (1996).

[3] D. Sun and O. Gang, J. Am. Chem. Soc. 133, 5252 (2011).

[4] R. T. Scarlett, M. T. Ung, J. C. Crocker, and T. Sinno, Soft Matter 7, 1912 (2011).

[5] D. Frenkel and D. J. Wales, Nat. Mater. 10, 410 (2011).

[6] F. Martinez-Veracoechea, B. Mladek, A. Tkachenko, and D. Frenkel, Phys. Rev. Lett. 107, $10(2011)$.

[7] R. Dreyfus, M. E. Leunissen, R. Sha, A. Tkachenko, N. C. Seeman, D. J. Pine, and P. M. Chaikin, Phys. Rev. E 81, 1 (2010).

[8] S. Sacanna, W. T. M. Irvine, P. M. Chaikin, and D. J. Pine, Nature 464, 575 (2010).

[9] K.-T. Wu, L. Fenga, R. Shab, R. Dreyfusc, A. Y. Grosberga, N. C. Seemanb, and P. M. Chaikin, P. Natl. Acad. Sci. USA 109, 1873118736 (2012).

[10] Y. Wang, Y. Wang, D. R. Breed, V. N. Manoharan, L. Feng, A. D. Hollingsworth, M. Weck, and D. J. Pine, Nature 491, 51 (2012).

[11] F. Vargas Lara and F. W. Starr, Soft Matter 7, 2085 (2011).

[12] P. E. Theodorakis, C. Dellago, and G. Kahl, J. Chem. Phys. 138, 025101 (2013).

[13] H. Tang, R. Deschner, P. Allen, Y. Cho, P. Sermas, A. Maurer, A. D. Ellington, and C. G. Willson, J. Am. Chem. Soc. 134, 15245 (2012).

[14] F. Martinez-Veracoechea and M. E. Leunissen, Soft Matter 9, 3213 (2013).

[15] R. J. Macfarlane, B. Lee, H. D. Hill, A. J. Senesi, S. Seifert, and C. A. Mirkin, P. Natl. Acad. Sci. USA 106, 10493 (2009).

[16] L. Di Michele and E. Eiser, Phys. Chem. Chem. Phys. 15, 3115 (2013).

[17] S. Tindemans and B. Mulder, Phys. Rev. E 82, 1 (2010).

[18] J. C. Crocker, Nature 451, 528 (2008).

[19] S. V. Buldyrev, G. Malescio, C. A. Angell, N. Giovambattista, S. Prestipino, F. Saija, H. E. Stanley, and L. Xu, J. Phys.: Condens. Mat. 21, 504106 (2009).

[20] J. S. Høye, E. Lomba, and N. G. Almarza, Mol. Phys. 107, 321 (2009).

[21] P. Akcora, H. Liu, S. K. Kumar, J. Moll, Y. Li, B. C. Benicewicz, L. S. Schadler, D. Acehan, A. Z. Panagiotopoulos, V. Pryamitsyn, et al., Nat. Mater. 8, 354 (2009).

[22] V. Pryamtisyn, V. Ganesan, A. Z. Panagiotopoulos, H. Liu, and S. K. Kumar, J. Chem. Phys. 131, $221102(2009)$. 
[23] V. Manoharan, in Perimeter Institute Colloquium Series (2010), URL http://streamer. perimeterinstitute.ca/Flash/10438c30-a81a-4d44-9723-2f0de25775ab/index.html.

[24] M. W. Matsen, J. Phys.: Condens. Mat. 14, R21 (2002).

[25] M. W. Matsen, in Soft Matter Vol. 1: Polymer Melts and Mixtures, edited by G. Gompper and M. Schick (Wiley-VCH Verlag, Weinheim, Germany, 2005), vol. 1 of Soft Matter, chap. 2.

[26] G. H. Fredrickson, The Equilibrium Theory of Inhomogeneous Polymers (Oxford University Press, New York, 2006).

[27] A. J. Archer, Phys. Rev. E 78, 031402 (2008).

[28] A. J. Archer, C. Ionescu, D. Pini, and L. Reatto, J. Phys.: Condens. Mat. 20, 415106 (2008).

[29] The theory and simulation of Pryamtisyn et al. [22] is more appropriate for a detailed understanding of the polymer stabilized colloidal case. Specifically, the isotropic interactions between particles in this case become anisotropic as the colloids aggregate.

[30] P. L. Biancaniello, A. J. Kim, and J. C. Crocker, Phys. Rev. Lett. 94, 058302 (2005).

[31] W. B. Rogers and J. C. Crocker, P. Natl. Acad. Sci. USA 108, 1568715692 (2011).

[32] Mirkin et al. [1] use colloids of a $13 \mathrm{~nm}$ diameter in water. A rough value for the diameter of a water molecules is $2.74 \AA$. A quantitatively realistic value for $\alpha$ for this case should then be about $10^{-6}$ which is not computationally feasible. Instead, qualitative trends are investigated in this work.

[33] K. von Konigslow, Master's thesis, University of Waterloo, Waterloo, Ontario, Canada (2012), URL http://hdl.handle.net/10012/7037.

[34] M. W. Matsen and F. S. Bates, Macromolecules 29, 7641 (1996).

[35] R. B. Thompson, T. Jebb, and Y. Wen, Soft Matter 8, 9877 (2012).

[36] R. B. Thompson, Phys. Rev. E 73, 020502(R) (2006).

[37] R. B. Thompson, Phys. Rev. E 74, 041501 (2006).

[38] R. B. Thompson, K. O. Rasmussen, and T. Lookman, J. Chem. Phys. 120, 31 (2004). 